\title{
Predictors of Post-mastectomy Breast Reconstruction in Kenya
}

\author{
Stephen Gichuru, Thomas Kedera, Joseph Wanjeri, Peter Ndaguatha
}

University of Nairobi

Correspondence to: Dr. Stephen Gichuru, PO Box 30197-00100, Nairobi; email: mwangidrsteve@gmail.com

\begin{abstract}
Background: Breast reconstruction following mastectomy has mental and physical health benefits. Several patient-centered factors predict the rate of breast reconstructions. We investigated the rates and predictors of breast reconstruction in postmastectomy patients diagnosed with breast cancer at a teaching and national referral center. Methods: A retrospective case-control study involving post-mastectomy females diagnosed with breast cancer from 2014 to 2018. Logistic regression evaluated potential predictors of breast reconstruction. Results: 312 women had mastectomies during the 5-year study period: 9 (2.88\%) had breast reconstructionall breast reconstructions were autologous (latissimus flap). The rate of reconstruction increased over the study period, then dropped in 2018. Factors significantly affecting the rate of breast reconstruction included
\end{abstract}

\section{Introduction}

Breast reconstruction (BR) not only brings shape to a breast it also restores a woman's identity, attractiveness and sexual desirability, and hence affects their quality of life (1). Following the procedure, patients experience several complications; nonetheless, rates of breast cancer recurrence appear not to be a direct consequence of breast reconstruction. The multivariate mechanisms that would essentially elucidate recurrence in vitro seem not to translate clinically (2). Thus, breast cancer patients, who have undergone a mastectomy, should have a BR recommendation for maximal management of patients (3).

There are, however, predictors that affect the rates of BR postmastectomy $(1,4)$. The reasons stated for getting a BR are physician-associated, patient-associated, and healthcare financing $(4,5)$. Data is limited on postmastectomy breast reconstruction (PMBR) from the African countries. In developing countries, the procedure is unpopular in the full rehabilitation of the patient. Further accreditation may arise from few plastic surgeons and heavy workload, and not many patients know about their rehabilitation options postmastectomy (1).

In Kenya, no data has been published describing the patient age and insurance coverage. Conclusion: In this hospital-based study, the rate of post-mastectomy breast reconstruction is low, despite the procedure being an essential component of breast cancer care. Age and insurance coverage are predictors for breast reconstruction.

Keywords: Breast reconstruction, Rates, Hospitalbased, Predictors

Ann Afr Surg. 2020; 17(1):16-20

DOI: http://dx.doi.org/10.4314/aas.v17i1.5

Conflicts of Interest: None

Funding: None

(C) 2020 Author. This work is licensed under the Creative Commons Attribution 4.0 International License.

limitations and rates of breast reconstruction following mastectomy. In this study, we aimed to determine the predictors for breast reconstruction.

\section{Materials and Methods}

This retrospective hospital-based, case-control study made use of the resources of the Kenyatta National Hospital database. The population for this study targeted opt-in female patients 18 years and above and postmastectomy patients diagnosed with breast cancer between 2014 to 2018 . The cases and controls matched because they underwent a mastectomy procedure; the cases had breast reconstruction while the controls did not. The primary outcome was breast reconstruction defined by these codes for implant-based reconstruction (IBR) and autologous reconstruction (AR).

We investigated the following patient predictor variables: age, ethnicity, medical comorbidities, insurance status, income, marital status, religion, level of education, postoperative need for radiotherapy and chemotherapy, and contralateral prophylactic mastectomy. We entered all information in a computer and analysed it using SPSS version 25 (Inc, Chicago, IL, USA) for Windows and MacOS. We obtained consent for the study from 
Kenyatta National Hospital University of Nairobi Ethics and Research Committee (UP600/08/2018).

\section{Results}

\section{Sample characteristics}

Mastectomy patients. The mean age of patients at the time of mastectomy was $46.79(20.71-72.87)$ years, and the modal age 40 years. Most patients (58.9\%) were married, $26 \%$ were single, $8 \%$ widowed, $7 \%$ separated, and $1 \%$ divorced. Only $2.74 \%$ of patients had a bilateral mastectomy. In our study, $98.2 \%$ of these mastectomy patients were Christians, and only $1.8 \%$ Muslims. Most mastectomy patients (84.4\%) were Bantus, 14.2\% Nilotes, and 1.4\% Cushites. The highest level of education for most patients $(43.8 \%)$ was secondary education, next was primary (30.1\%), tertiary (19.2\%) and $6.8 \%$ had no formal education. We looked at the 10-year survival rate of the patients using the Charlson comorbidity index. Most patients (48.4\%) had a $90 \%$ probability of surviving (Fig. 1).

Almost $90 \%$ of patients had insurance: most (96.9\%) could afford paying premiums for the National Health Insurance Fund (NHIF). Notably, most of the patients who underwent mastectomy (68\%) had another mode of payment besides the insurance.

The modal breast size was 40D (27.9\%). Modified radical mastectomy (MRM) was the most common at $72.6 \%$. Most patients needed further treatment: $55.3 \%$ needed radiotherapy, while $89 \%$ had to undergo chemotherapy. A few patients (2.7\%) underwent contralateral prophylactic mastectomy. We included all mastectomy patients in the study.

Breast reconstruction patients. The cases included nine female patients with a mean age of 34.33 (16.00-52.66) years; modal age was 35, and all were Christians. Eight of these patients were married and Bantu. The highest level of education was tertiary, for seven patients; two patients had reached secondary school. For physical characteristics, the modal breast size was 40D (three patients). Using the Charlson comorbidity index, seven patients had a $90 \%$ probability of surviving, one a probability of $77 \%$ and one a $0 \%$ probability. Eight

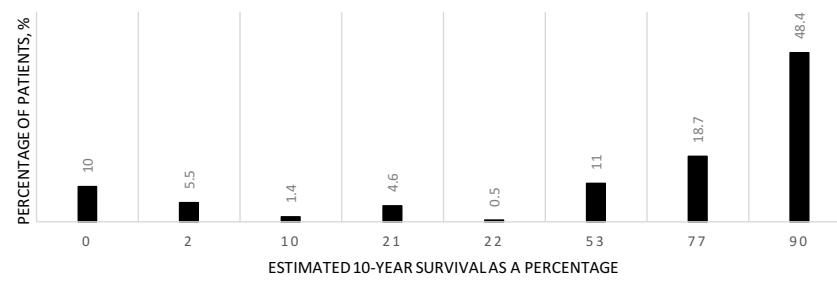

Figure 1. The Charlson comorbidity index for mastectomy patients patients had insurance cover: six patients could afford the NHIF and two had private insurance. For the breast reconstruction patients, six patients had no other mode of payment other than insurance cover, one patient was financed by the community, and one paid out of pocket. Trend in breast reconstruction. The $\mathrm{KNH}$ database yielded data for 312 women with codes for mastectomy. In this group, nine women had breast reconstruction after mastectomy, for an overall reconstruction rate of $2.88 \%$. The rate increased annually over the study period, reaching a peak of $10.81 \%$ in 2017 and then sharply dropping to $1.41 \%$ in 2018 , coinciding with an increase in the number of mastectomies from 37 in 2017 to 71 in 2018. The average number of mastectomies per year was 57. Figure 2 shows the five-year trend using a polynomial trend line, which is used when data fluctuates in linear regression $\left(R^{2}=1\right.$, the perfect fit). All breast reconstructions were autologous (latissimus flap). Figure 3 shows the pattern of immediate versus delayed breast reconstruction and includes polynomial trendlines to demonstrate a progressive increase in immediate breast reconstructions over time compared with a predicted decline delayed breast reconstruction ( $R^{2}=1$, the perfect fit).

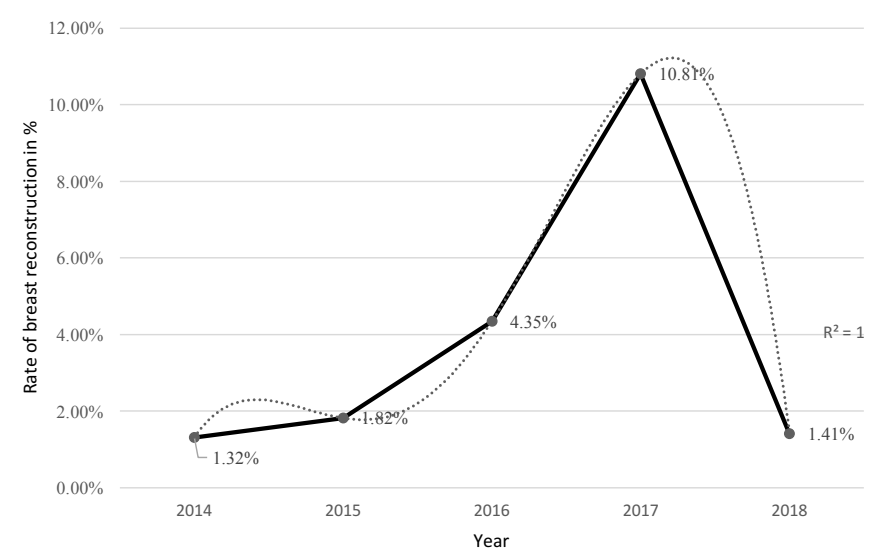

Figure 2. Postmastectomy breast reconstruction rate by fiscal year of mastectomy.

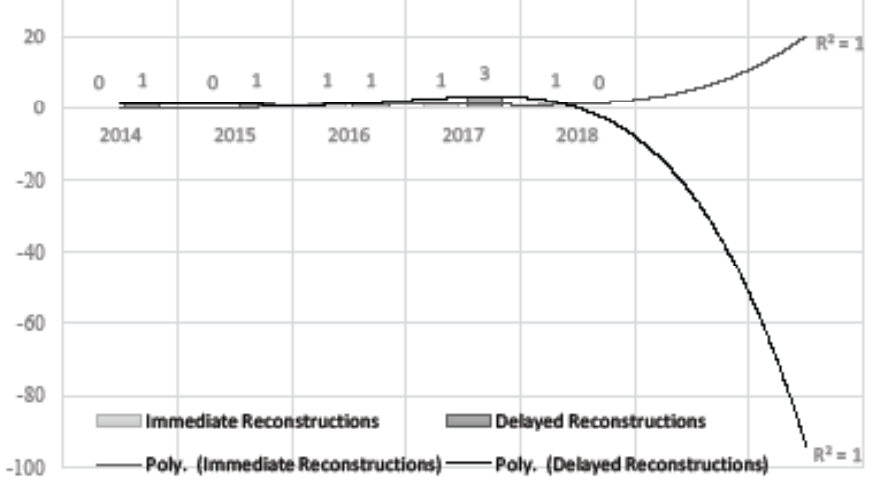

Figure 3. Number of immediate and delayed breast reconstructions by fiscal year of mastectomy 
Binary logistic regression. In the binary logistic regression, breast reconstruction was the primary dependent variable and it had a dichotomous response (No or Yes). The best-fitted model showed that insurance cover and age (Table 1) accounted for $52.3 \%$ of the variance of having undergone breast reconstruction. HosmerLemeshow goodness-of-fit test was greater than 0.05 (at 0.972), showing that the fitted model had a good fit.

Age. Age had a negative beta coefficient of -0.102 , meaning that undergoing breast reconstruction becomes less likely as the predictor age increases. We coded age as a binary variable: below 50 years and 50 and above years. WHO defines old age arbitrarily in Africa as $\mathbf{5 0}$ years, therefore in our study we used 50 years as the lower age threshold for considering older patients, and anyone below 50 was considered "young" (6). Among the young women, the modal cohort was 26 to 35 years of age at $44 \%$ (Fig. 4 ).

Insurance. Insurance coverage had a negative beta coefficient of -4.836 , indicative of a negative association.

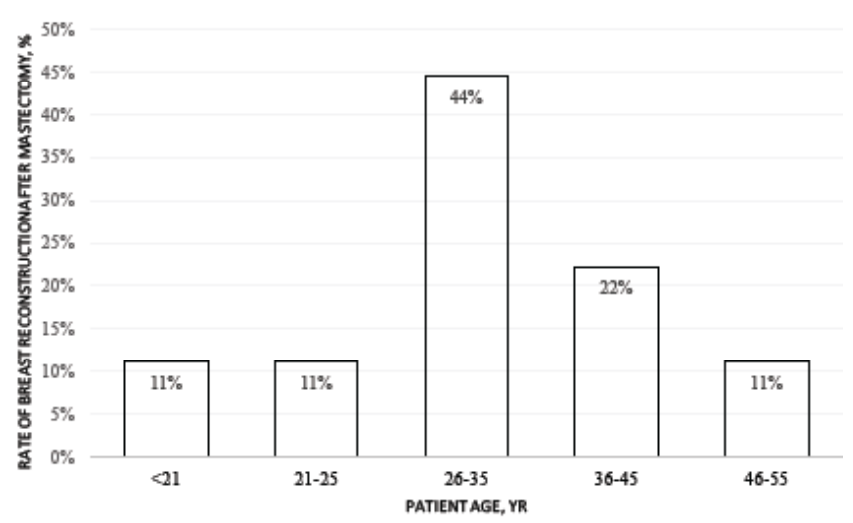

Figure 4. The rate of breast reconstruction postmastectomy, by patient age.

Therefore, breast reconstruction following mastectomy becomes less likely for patients whose insurance does not cover breast reconstruction following mastectomy (Table 1).

Table 1. Binary logistic regression analysis of factors affecting incidence of post-mastectomy breast reconstruction

\begin{tabular}{lll}
\hline Factor & Odds ratio $(95 \% \mathrm{Cl})$ & $\mathrm{p}$ value \\
\hline Age & $0.175(0.004-0.345)$ & $<0.001$ \\
Insurance coverage & $0.008(0.001-0.061)$ & $<0.001$ \\
\hline
\end{tabular}

\section{Discussion}

From our hospital-based study, $2.88 \%$ of postmastectomy patients underwent BR in five years. This rate is substantially lower than in Canadian hospital-based studies on BR that range from $3.8 \%$ to $21 \%$. The same holds for tertiary care centers in the United States, whereby the rates of BR go up to $42 \%(4,7)$. In Asia, a similar study showed a rate of $24.3 \%$ while in China the rate remains stable at $3.5-4.5 \%$ over the past 15 years $(8,9)$. Our study focused on a tertiary public hospital; a comparative study in a private hospital in Kenya has yet to be done.

We demonstrate that the proportion of unilateral mastectomy is high, whereas bilateral mastectomy remains low. This proportional difference is unique as trends show the rate of bilateral mastectomy is increasing, whereas unilateral mastectomy rates are decreasing (10). Our five-year trend shows a gradual increase in the rate of BR as in the case in Canada, USA, China, and Singapore (7-9). This finding may suggest a rise in the awareness of BR. With time, more women have increased access to information about BR from the internet.

All breast reconstructions were autologous (latissimus flap). This trend is unique to other countries where both implant and autologous based surgery utility is present. The overall trend is an increase in implantbased BR since most patients are opting for immediate breast reconstruction. We state that there is a progressive increase in immediate breast reconstructions over time compared to a predicted decline delayed BR in our tertiary center similar to other hospital-based studies $(7-9,11)$. Further research into the determinants of timing of the BR needs to be explored, as our study design limited the ability to capture the influences of the trend at our hospital.

Age and insurance coverage of the patient were the only significant factors affecting BR in our setup. Age appears to be a persistent factor that affects the likelihood of undergoing $\mathrm{BR}$ in this and other studies. Younger patients are more likely to have the procedure done. Young patients are less likely to undergo autologous reconstruction due to more scar formation at the donor site. Patients older than fifty have more complications; however, reconstruction is not a contraindication in this age group $(4,5,7)$.

Insurance coverage and higher socioeconomic status of the community likely facilitate access to BR, as seen in our set up $(5,12)$. Yang et al. stated that the possibility that patients with non-private health insurance are more likely to undertake the delayed BR; in the USA, insurance status is strongly associated with receipt of immediate BR especially for those with privately bought insurance (13). The NHIF covers all surgical procedures of therapeutic and preventive value (14). Surgeons do not recommend palliative reconstructive surgery as 
part of the management of breast cancer patients (3). Moreover, there is no documentation of whether NHIF covers BR as a cosmetic or a therapeutic procedure.

Income did not appear as a significant factor in BR in our patients. Our finding contradicts the expected as several studies predict household income as an influencer of the rate of PMBR. In a non-universal health care system, women with higher income background were more likely to have had PMBR $(12,15)$.

We found no association between the breast size of a patient before mastectomy and the likelihood of getting breast reconstruction. The role of preoperative breast size in choosing the aptest BR option would have led to the conclusion that there would be an association $(16,17)$.

This study found no association between the breast size of a patient before mastectomy and the likelihood of getting breast reconstruction. The role of preoperative breast size in choosing the most suitable BR option would have led to the conclusion that there would be an association $(16,17)$.

Based on our study, the frequency of breast cancer from highest tolowest among the black Kenyan tribes is Bantus, Nilotes, and Cushites (18). The ethnic background of the patient affects the presentation and time of diagnosis of disease. Furthermore, ethnicity tended to determine the procedure performed. African American women had increased rates of bilateral implant-based reconstruction while unilateral flap-based reconstructions rates were common among Hispanic patients (19). In our study, we established no association between the ethnic group and BR despite most of our study population being Bantu women.

Majority of our breast reconstructed patients have a tertiary education. Higher levels of educations are associated with an increased ability to comprehend the procedure use and outcomes preoperatively as offered by the involved surgical stakeholders. This comprehension has been shown to lead to increased odds of undergoing $\operatorname{BR}(12,20)$. However, we did not find any association in our current study.

In a review of literature, researchers include marital status in the analysis of hinderances of BR (12). However, other studies have shown that marital status is not an explicit predictor for PMBR $(15,21)$. The later was replicated in our study.

We found no association between the comorbidities and BR with the aid of the Charlson comorbidity index (CCI). In a study looking at the relationship between comorbidities and quality of life, the authors established that comorbidities did not influence the choice of surgery with or without BR (22). In terms of shorter overall survival, prognostic factors such as lymph node metastasis (the most significant), the grade of disease, size of tumor and multifocality of the disease have yet to be correlated (23).

Further therapy is a hindrance to PMBR where radiotherapy is associated with $\mathrm{AR}(4,24)$. In our hospital, we found no associations between the need for chemotherapy and radiotherapy as negative predictors to PMBR.

\section{Conclusion}

In this hospital-based study, the rate of post-mastectomy breast reconstruction is low in the tertiary referral health center in Kenya despite the procedure being an essential component of breast cancer care. Efforst must be increased to enhance awareness among surgical stakeholders and the general population on the surgery. Age and insurance coverage are predictors for breast reconstruction.

\section{Acknowledgements}

We thank Ms. Janet Ndung'u and Mr. Branley Ope for helping with data collection.

\section{References}

1. Olaitan PB. Breast cancer management: A review of the role of plastic and reconstructive surgery. Niger J Plast Surg. $2011 ; 1 ; 7(2): 48-58$.

2. Brett EA, Aitzetmüller MM, Sauter MA, et al. Breast cancer recurrence after reconstruction: Know thine enemy. Oncotarget. 2018; 12;9(45):27895-906.

3. E Wata D, Osanjo G, Oluka M, et al. Predictors of Breast cancer treatment outcomes in Kenyan women. Afr J Pharmacol Ther. 2013; 1;2:109.

4. Platt J, Baxter N, Zhong T. Breast reconstruction after mastectomy for breast cancer. CMAJ Can Med Assoc J. $2011 ; 13 ; 183(18): 2109-16$.

5. Morrow M, Li Y, Alderman AK et al. Access to breast reconstruction and patient perspectives on decision making. JAMA Surg. 2014; 149(10):1015-21.

6. Kowal P, Peachey K. Indicators for the minimum data set project on ageing: A critical review in Sub-Saharan Africa. US National Institute on Aging; 2001. https://www.who.int/ healthinfo/survey/ageing_mds_report_en_daressalaam. pdf

7. Karunanayake M, Bortoluzzi P, Chollet A, et al. Factors influencing the rate of post-mastectomy breast reconstruction in a Canadian teaching hospital. Plast Surg. $2017 ; 25(4): 242-8$.

8. Huang N-S, Quan C-L, Ma L-X-X, et al. Current status of breast reconstruction in China: An experience of 951 breast reconstructions from a single institute. Gland Surg. 2016; $5(3): 278$.

9. Sim N, Soh $\mathrm{S}$, Ang $\mathrm{CH}$, et al. Breast reconstruction rate 
and profile in a Singapore patient population: A national university hospital experience. Singapore Med J. 2018; 59(6):300.

10. Nelson JA, Lee IT, Disa JJ. The functional impact of breast reconstruction: An overview and update. plast reconstr surg glob open. 2018; 6(3). https://www.ncbi.nlm.nih.gov/ pmc/articles/PMC5908499/

11. Lam TC, Winch CJ. What would women choose when given a choice in breast reconstruction? Plast Reconstr Surg Glob Open. 2016; 4(9). https://www.ncbi.nlm.nih.gov/pmc/ articles/PMC5055028/

12. Soni SE, Lee MC, Gwede CK. Disparities in use and access to postmastectomy breast reconstruction among African American women: A targeted review of the literature. Cancer Control. 2017; 24(4):1073274817729053.

13. Yang RL, Newman AS, Lin IC, et al. Trends in immediate breast reconstruction across insurance groups after enactment of breast cancer legislation. Cancer. 2013; $1 ; 119(13): 2462-8$.

14. National Health Insurance Fund (NHIF). comprehensive medical insurance scheme for civil servants \& disciplined services hand book. NHIF; 2012. http://www.nhif. or.ke/healthinsurance/uploads/manuals/civil_servants handbook.pdf

15. Ballard TNS, Kim Y, Cohen WA, et al. Sociodemographic predictors of breast reconstruction procedure choice: Analysis of the mastectomy reconstruction outcomes consortium study cohort. Plast Surg Int. 2015; 2015:150856.

16. Colwell AS, Christensen JM. Nipple-sparing mastectomy and direct-to-implant breast reconstruction. Plast Reconstr Surg. 2017; 140(5S Advances in Breast Reconstruction): 44S-50S.
17. Schwartz J-CD, Skowronski PP. Total single-stage autologous breast reconstruction with free nipple grafts. Plast Reconstr Surg Glob Open. 2016; 3(12). https://www.ncbi.nlm.nih. gov/pmc/articles/PMC4727696/

18. Korir A, Wang EY, Sasieni P, et al. Cancer risks in Nairobi (2000-2014) by ethnic group. Int J Cancer. 2017; 140(4):788-97.

19. Rodby KA, Danielson KK, Shay E, et al. Trends in breast reconstruction by ethnicity: An institutional review centered on the treatment of an urban population. Am Surg. 2016; 82(6):497-504.

20. Hasak JM, Myckatyn TM, Grabinski VF, et al. Stakeholders' perspectives on postmastectomy breast reconstruction: Recognizing ways to improve shared decision making. Plast Reconstr Surg Glob Open. 2017; 5(11). https://www.ncbi. nlm.nih.gov/pmc/articles/PMC5732675/

21. Awan BA, Samargandi OA, Alghamdi HA, et al. The desire to utilize postmastectomy breast reconstruction in Saudi Arabian women. Saudi Med J. 2015; 36(3):304-9.

22. Fu MR, Axelrod D, Guth AA, et al. Comorbidities and quality of life among breast cancer survivors: A prospective study. J Pers Med. 2015; 5(3):229-42.

23. Akinyi MO, Dan K, Daniel OK, et al. Correlation between receptor status and presence of axillary lymph node metastasis in breast cancer in Kenya. Ann Afr Surg. 2019; 16(2). https://www.ajol.info/index.php/aas/article/view/ 188641

24. Cohen O, Lam G, Choi M, et al. Does the timing of chemotherapy affect post-mastectomy breast reconstruction complications? Clin Breast Cancer. 2017; 17(4):307-15. 